\title{
A 3x3 Switch Exploiting an Optical Vortex Beam Emitter based on a Silicon Three-Grating Microring
}

\author{
Mirco Scaffardi ${ }^{1}$, Muhammad N. Malik ${ }^{1,2}$, Emma Lazzeri $^{2}$, Charalambos Klitis ${ }^{3}$, Laura Meriggi ${ }^{3}$, \\ Ning Zhang ${ }^{3}$, Marc Sorel ${ }^{3}$, Antonella Bogoni ${ }^{2}$ \\ 1. CNIT, via G. Moruzzi 1, 56124, Pisa \\ 2. Scuola Superiore Sant'Anna, Via Moruzzi 1, 56124 Pisa, Italy \\ 3. University of Glasgow, Oakfield Avenue, Glasgow G12 8LT, UK \\ Corresponding author e-mail address: mirco.scaffardi@cnit.it
}

\begin{abstract}
A silicon three-grating microring is proposed and characterized as a device enabling $3 \times 3$ optical switching based on orbital angular momentum and wavelength. Bit error rate measurements show penalties $<1 \mathrm{~dB}$ for OOK traffic up to $20 \mathrm{Gbaud}$.

OCIS codes: (130.3120) Integrated optics devices; (130.4815) Optical switching devices.
\end{abstract}

\section{Introduction}

The optical switching can offer effective solutions to overcome the scalability issues of current electrical interconnection networks, which are limited by power consumption, power dissipation and footprint problems [1]. Interconnection networks scalability can be enhanced with multiple switching domains. The traditional envisioned switching domains are space, wavelength, and time [2]. The orbital angular momentum (OAM) of light can represent a further domain to improve scalability and performance of the multilayer interconnection networks, since beams with different OAM order are orthogonal, i.e. they can propagate together ideally without crosstalk [3]. A reconfigurable 2x2 OAM optical switch has been demonstrated based on bulk spatial light modulators (SLMs)[4]. Integrated devices such as microrings with super-imposed grating structures [5], circular grating couplers cascaded to star couplers [6] and hybrid 3D integrated circuits [7] have been demonstrated as a compact alternative to the OAM light generation. Integrated devices are attractive for the small form factor (i.e. tens of micrometers) and lowpower and fast tunability (i.e. $\mu$ s timescale and tens of $\mathrm{mW}$ with thermal tuning). In [8] we proposed a switching architecture exploiting OAM and wavelength as switching domains based on integrated OAM emitters.

Here we propose and characterize, for the first time, a Silicon three-grating microring as device enabling $3 \times 3$ optical switching. The switch exploits simultaneously the OAM and wavelength as switching domains, allowing full configurability without blocking states.

\section{Working principle and setup}

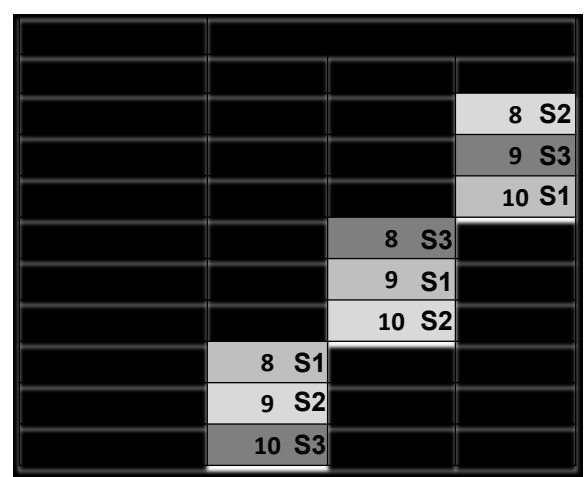

(a)

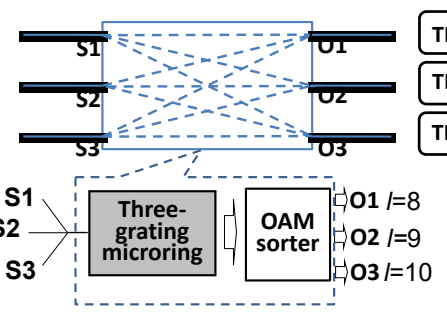

(b)

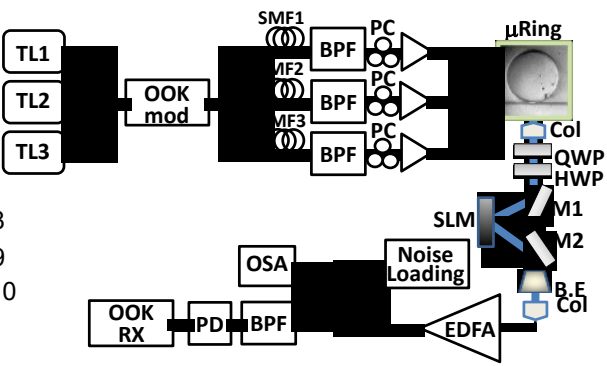

(c)

Fig. 1 (a) Mapping of the OAM mode order $l$ emitted by the microring on the three gratings vs. wavelength $\lambda i$ of the resonance peaks. (b) Scheme of a 3x3 OAM-based switch exploiting a three-grating microring and an OAM mode sorter. (c) Experimental setup for the characterization.

A 3x3 OAM-based switch can be implemented by exploiting a Silicon microring with three overlapped gratings [5]. The microring with radius $29 \mu \mathrm{m}$, fabricated by the University of Glasgow, has three overlapped gratings with different number of grating elements $q_{i}$. Each grating emits an OAM mode of order $l_{i}$ depending on the resonance peak (free spectral range $\sim 3 \mathrm{~nm}$ ) at which the input wavelength is set (see Fig. 1 (a)). Fixing the input wavelength, the difference among the orders of the OAM modes emitted simultaneously by the three microrings is $\Delta q=q_{2^{-}} q_{1}=q_{3^{-}}$ $q_{2}=3$. According to Fig. 1 (a), a set of three OAM modes $(l=8,9,10)$ can be selected to build a $3 \times 3$ OAM switch, as detailed in Fig. 1 (b). The switch is composed by the three-grating microring and an OAM mode sorter. The three- 
grating microring emits a set of three OAM modes with order depending on the wavelength of the three input signals $\mathrm{S} 1, \mathrm{~S} 2$, and S3. Three different disjoined sets of three wavelengths can be assigned to each input $\mathrm{Si}$ in such a way that, by proper selection of the wavelength, the input light can be converted to one of the OAM modes of order 8,9 and 10. A passive OAM mode sorter allows the routing of the signal coming from each input $\mathrm{Si}$ to a different spatial direction depending on the order of the incoming OAM mode, which therefore identifies the switch output port [8]. Since the triplet of wavelength associated to S1, S2 and S3 is different, all the input signals can be directed to the same output port without interference. The experimental setup is shown in Fig. 1 (c). The input signals are generated from three tunable lasers at different wavelength fed into a Mach-Zehnder amplitude modulator driven by an electrical PRBS $2^{31}-1$. The signal replica are decorrelated with single mode fibre spans (SMFi). EDFAs sets the power at the microring input at $25 \mathrm{dBm}$ in order to compensate for the coupling losses. The signal is fed into the microring with a tapered fiber with spot size $5 \mu \mathrm{m}$. Each signal S1, S2 and S3 emitted from the microring propagates collimated in free space along a direction orthogonal to the chip plane. A SLM selects an OAM mode and converts it back to a Gaussian beam for fibre coupling. The OAM mode conversion operation is performed independently of the signal wavelength. The signal is then amplified, loaded with noise and filtered (BPF) before being received. The spectrum is monitored with an optical spectrum analyser (OSA).

\section{Experimental results}

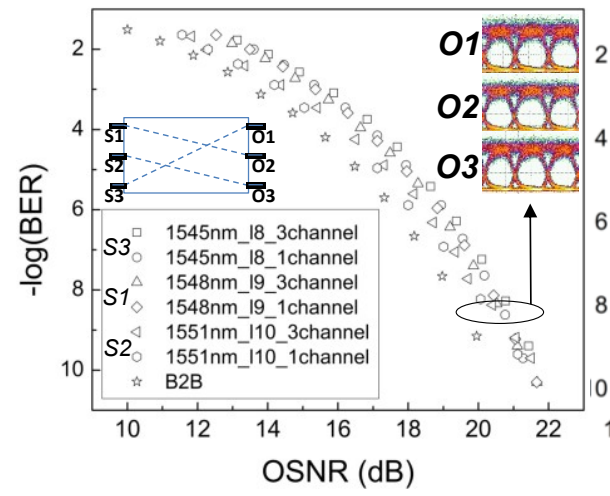

(a)

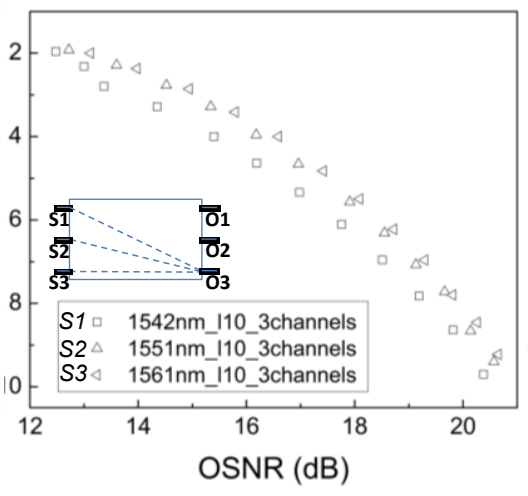

(b)

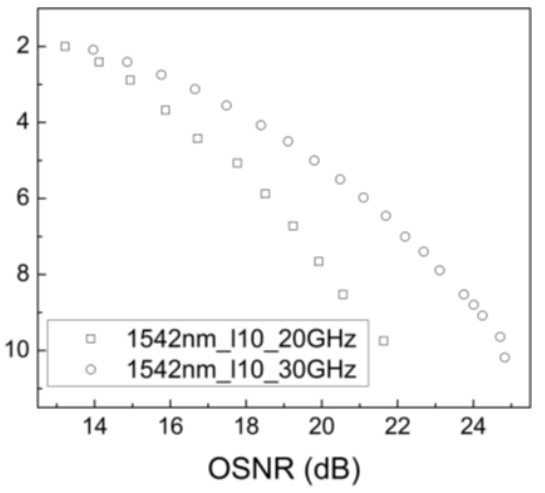

(c)

Fig. 2 (a) BER vs. OSNR for switch configured as follows: S1 to O2, S2 to O3, S3 to O1. Output eye diagram in the inset. (b) BER vs. OSNR for switch configured as follows: S1 to O3, S2 to O3, S3 to O3. (c) Comparison of BER vs. OSNR at 20 and 30 Gbaud.

Performance is measured at 20 Gbaud. Fig. 2 (a) shows the bit error rate (BER) vs. optical signal-to-noise ratio (OSNR) when each input signal is directed to a different output port, i.e. S1 directed to O2, S2 to O3, S3 to O1. At $\mathrm{BER}=10^{-9}$ the OSNR sensitivity difference among the output signals is $<0.5 \mathrm{~dB}$ when all the three input channels are present. There is no performance degradation with respect to the case where a single channel is present at the input, i.e. the microring performance does not depend on the number of input signals. The penalty with respect to the backto-back, measured by replacing the microring with a filter of the same $3 \mathrm{~dB}$ bandwidth $(\sim 0.2 \mathrm{~nm})$, is $<1 \mathrm{~dB}$. The three insets show an open eye diagram for the three signals at the microring output. Fig. 2 (b) shows the BER vs. OSNR when all the input signals are sent to the same output port. OSNR sensitivity difference $<0.5 \mathrm{~dB}$ is measured. By increasing the baud rate up to 30 Gbaud, still error-free performance can be obtained, with an additional penalty of $3.5 \mathrm{~dB}$ (see Fig. 2 (c)).

This work has been done within the Project ROAM-Revolutionizing optical fiber transmission and networking using the orbital angular momentum of light (Call ID: H2020-ICT-2014-1; topic: ICT-06-2014; funding scheme: RIA; contract number: 645361).

\section{References}

[1]H. Cho, et al., "Power comparison between high-speed electrical and optical interconnects for interchip communication," J. Light. Tech., v. 22, n. 9, p. 2021, 2004.

[2]I. Cerutti, et al., "Power and scalability analysis of multi-plane optical interconnection networks", IET Optoelect., v. 6, n. 4, p. 192, 2012.

[3]J. Wang, et al., "Terabit free-space data transmission employing orbital angular momentum multiplexing", Nat. Photonics, v. 6, p. 488, 2012.

[4]N. Ahmed, et al., "Reconfigurable $2 \times 2$ orbital angular momentum based optical switching of 50 -Gbaud QPSK channels", Optics Express, v.

22, n. 1, p. 756, 2014.

[5]Q. Xiao, et al., "Generation of photonic orbital angular momentum superposition states using vortex beam emitters with superimposed gratings", Optics Express, v. 24, n. 4, 3168, 2016.

[6]N. K. Fontaine et al., "Efficient multiplexing and demultiplexing of free-space orbital angular momentum using photonic integrated circuits", OFC, OTu1I.2, 2012.

[7]C. Quin et al., "Demonstration of Orbital Angular Momentum State Conversion using Two Hybrid 3D Photonic Integrated Circuits", OFC, Th4A, 2014.

[8] M. Scaffardi et al., "A Silicon Microring Optical 2x2 Switch Exploiting Orbital Angular Momentum for Interconnection Networks up to 20Gbaud", IEEE Journal of Light. Techn., published on Nov. 17, 2016. 